\title{
Approximating MAX SAT by moderately exponential and parameterized algorithms
}

\author{
Bruno Escoffier ${ }^{\mathrm{a}, \mathrm{b}, *}$, Vangelis Th. Paschos ${ }^{\mathrm{c}, \mathrm{d}}$, Emeric Tourniaire ${ }^{\mathrm{c}}$ \\ a Sorbonne Universités, UPMC, LIP6, F-75005, Paris, France \\ b CNRS, UMR 7606, LIP6, F-75005, Paris, France \\ c PSL Research University, Université Paris-Dauphine, LAMSADE, CNRS, UMR 7243, France \\ d Institut Universitaire de France, France
}

\section{A R T I C L E I N F O}

\section{Article history:}

Received 29 October 2012

Accepted 21 October 2014

Available online 13 November 2014

\section{Keywords:}

Maximum satisfiability

Exponential time algorithms

Approximation algorithms

\begin{abstract}
A B S T R A C T
We study approximation of the MAX SAT problem by moderately exponential algorithms. The general goal of the issue of moderately exponential approximation is to catch-up on polynomial inapproximability, by providing algorithms achieving, with worst-case running times importantly smaller than those needed for exact computation, approximation ratios unachievable in polynomial time. We develop several approximation techniques that can be applied to MAX SAT in order to get approximation ratios arbitrarily close to 1 .
\end{abstract}

(C) 2014 Elsevier B.V. All rights reserved.

\section{Introduction}

Optimum satisfiability problems are of great interest from both theoretical and practical points of view. Let us only note that several subproblems of MAX SAT and MIN SAT are among the first complete problems for many approximability classes $[1,16]$. On the other hand, in many fields (including artificial intelligence, database system, mathematical logic,...) several problems can be expressed in terms of versions of SAT [3].

Satisfiability problems have in particular drawn major attention in the field of polynomial time approximation as well as in the field of parameterized and exact solution by exponential time algorithms. Our goal in this paper is to develop approximation algorithms for MAX SAT with running times which, though being exponential, are much lower than those of exact algorithms, and with a better approximation ratio than the one achieved in polynomial time. This approach has already been considered for MAX SAT in [14,20], where interesting tradeoffs between running time and approximation ratio are given. It has also been considered for several other well known problems such as MINIMUM SET COVER [7,12], MIN COLORING [5,6], MAX INDEPENDENT SET and MIN VERTEX COVER [8], MIN BANDWIDTH [13,18], etc. Similar issues arise in the field of FPT algorithms, where approximation notions have been introduced, for instance, in [9,15]. In this article, we propose several improvements of the results of [14] and [20] using various algorithmic techniques.

Given a set of variables and a set of disjunctive clauses, MAX SAT consists of finding a truth assignment for the variables that maximizes the number of satisfied clauses. In what follows, we denote by $X=\left\{x_{1}, x_{2}, \ldots, x_{n}\right\}$ the set of variables and by $C=\left\{C_{1}, C_{2}, \ldots, C_{m}\right\}$ the set of clauses. Each clause consists of a disjunction of literals, a literal being either a variable

\footnotetext{
Research partially supported by the French Agency for Research under the DEFIS program "Time vs. Optimality in Discrete Optimization", ANR-09-EMER010, and by the project ALGONOW of the research funding program THALIS (co-financed by the European Social Fund - ESF and Greek national funds).

* Corresponding author.

E-mail addresses: bruno.escoffier@upmc.fr (B. Escoffier), paschos@lamsade.dauphine.fr (V.Th. Paschos), emeric.tourniaire@lamsade.dauphine.fr (E. Tourniaire).
} 
$x_{i}$ or its negation $\neg x_{i}$. A $\rho$-approximation algorithm for MAX SAT (with $\rho<1$ ) is an algorithm that finds an assignment satisfying at least a fraction $\rho$ of the maximal number of simultaneously satisfied clauses. The best known ratio guaranteed by a polynomial time approximation algorithm is $\alpha=0.796$ obtained in [2], while it is known that the problem (and even its restriction to instances where every clauses contain exactly three literals) is not approximable in polynomial time with ratio $7 / 8+\epsilon$, for any $\epsilon>0$, unless $\mathbf{P}=\mathbf{N P}$ [19]. A recent result [22] states that for any $\epsilon>0$, achieving a ratio $7 / 8+\epsilon$ is even impossible to get in time $O\left(2^{m^{1-\epsilon^{\prime}}}\right)$ for any $\epsilon^{\prime}>0$ unless the exponential time hypothesis fails. ${ }^{1}$ This latter result motivates the study of exponential time approximation algorithms.

Dealing with exact solution, [10] gives an exact algorithm working in time $O^{*}\left(1.3247^{m}\right)$ which is the best known bound so far wrt. the number of clauses. ${ }^{2}$ Dealing with the number $n$ of variables, the trivial $O^{*}\left(2^{n}\right)$ bound has not yet been broken down, and this constitutes one of the main open problems in the field of exact exponential algorithms. The parameterized version of MAX SAT consists, given a set of clauses $C$ and an integer $k$, of finding a truth assignment that satisfies at least $k$ clauses, or to output an error if no such assignment exists. In [10] the authors give a parameterized algorithm for MAX SAT running in time $O^{*}\left(1.3695^{k}\right)$.

Using the same notation as in [10], we say that a variable $x$ is an $(i, j)$-variable if it occurs positively in exactly $i$ clauses and negatively in exactly $j$ clauses. For any instance $C$ of MAX SAT, we will denote by OPT(C) (or OPT if no ambiguity occurs) an optimal set of satisfied clauses. Finally, we denote by $\alpha$ the ratio guaranteed by a polynomial time approximation algorithm. In general, $\rho$ will denote the approximation ratio of an algorithm, and, when dealing with exponential complexity, $\gamma$ will be the basis of the exponential term expressing it.

In order to fix ideas, let us give a first simple algorithm, useful to understand some of our results. In particular, it is one of the basic stones of the results in [14]. It is based upon the following two well known reduction rules.

Rule 1. Any clause containing an $(h, 0)$ - or a $(0, h)$-literal, $h \geqslant 1$, can be removed from the instance. This is correct because we can set this literal to TRUE or FALSE and satisfy the clauses that contain it.

Rule 2. Any (1,1)-literal can be removed too. Let $C_{1}=x_{1} \vee x_{2} \vee \cdots \vee x_{p}$ and $C_{2}=\neg x_{1} \vee x_{2}^{\prime} \vee \cdots \vee x_{q}^{\prime}$ be the only two clauses containing the variable $x_{1}$. If there exist two opposite literals $\ell$ and $\neg \ell$ in resp. $C_{1}$ and $C_{2}$, then we can satisfy both clauses by setting $x_{1}$ to TRUE and $\ell$ to FALSE and therefore we can remove these clauses. Otherwise, we can replace these clauses by $C=x_{2} \vee \cdots \vee x_{p} \vee x_{2}^{\prime} \vee \cdots \vee x_{q}^{\prime}$. The optimum in the initial instance is the optimum in the reduced instance plus 1 .

Algorithm 1. Build a tree as follows. Each node is labeled with a sub-instance of MAX SAT. The root is the initial instance. The empty instances (instances with no clauses) are the leaves. For each node whose label is a non-empty sub-instance, if one of the reductions above applies, then the node has one child labeled with the resulting (reduced) sub-instance. Else, a variable $x$ is arbitrarily chosen and the node has two children: in the first one, the instance has been transformed by setting $x$ to FALSE (the literals $x$ have been removed and the clauses containing the literal $\neg x$ are satisfied); in the second one $x$ is set to TRUE and the contrary happens. Finally, for both children the empty clauses are marked unsatisfied. Thus, each node represents a partial truth assignment. An optimal solution is a truth assignment corresponding to a leaf that has the largest number of satisfied clauses.

To evaluate the complexity of Algorithm 1, we count the number of leaves in the tree. Note that if the number of leaves is $T(n)$, then the algorithm obviously works in time $0^{*}(T(n))$. In the sequel, in order to simplify notations we will use $T(n)$ to denote both the number of leaves (when we express recurrences) and the complexity. We consider two ways to count the number of leaves. The former is by means of the variables. Each node has two children for which the number of remaining variables decreases by 1 . This leads to a number of leaves $T(n) \leqslant 2 \times T(n-1)$ and therefore $T(n)=0^{*}\left(2^{n}\right)$. The second is by means of the clauses. On each node, if the chosen variable is an $(i, j)$-variable, then the first child will have its number of clauses decreased by at least $i$ and the second child by at least $j$. The worst case, using the two reduction rules given above, is $i=1$ and $j=2$ (or $i=2$ and $j=1)$, that leads to $T(m)=T(m-1)+T(m-2)$ and therefore $T(m)=O^{*}\left(1.618^{m}\right)$.

In [14], the authors showed a way to transform any polynomial time approximation algorithm (with ratio $\alpha$ ) for MAX SAT into an approximation algorithm with ratio $\rho$ (for any $\alpha \leq \rho \leq 1$ ) and running time $O^{*}\left(1.618^{(\rho-\alpha)(1-\alpha)^{-1} m}\right.$ ). The basic idea of this algorithm is to build the same tree as in Algorithm 1 up to the fact that we stop the branching when enough clauses are satisfied. Then the $\alpha$-approximation polynomial algorithm is applied on the resulting sub-instances. As already mentioned, the best value of $\alpha$ is 0.796 [2]. Dealing with complexity depending on the number of variables, using local search techniques Hirsch [20] devises for any $\epsilon>0$ and any $k \geq 2$ a randomized algorithm that find with high probability a $(1-\epsilon)$ approximation for MAX- $k$-SAT (restriction of the problem to instances with clauses of size at most $k$ ) in time $O^{*}\left(\left(2-c_{\epsilon, k}\right)^{n}\right)$ where $c_{\epsilon, k}=2 \epsilon /(k(1+\epsilon))$. For MAX-2-SAT, the complexity is improved down to $0^{*}\left((2-3 \epsilon /(1+3 \epsilon))^{n}\right)$.

The paper is organized as follows. In Sections 2 and 3 we propose some improvements (for any ratio $\rho$ ) of the results of [14]. Fig. 1 illustrates the relationship approximation ratio-running time of the different methods we develop in these sections and compare them with the result in [14]. More precisely, in Section 2 two first results are presented: the first one

\footnotetext{
1 This hypothesis [21] says that MAX SAT where each clause has three literals is not solvable in subexponential time wrt. the number of variables.

2 We use the standard notation $O^{*}(f)$ to denote $f \times p(m+n)$ for some polynomial $p$.
} 


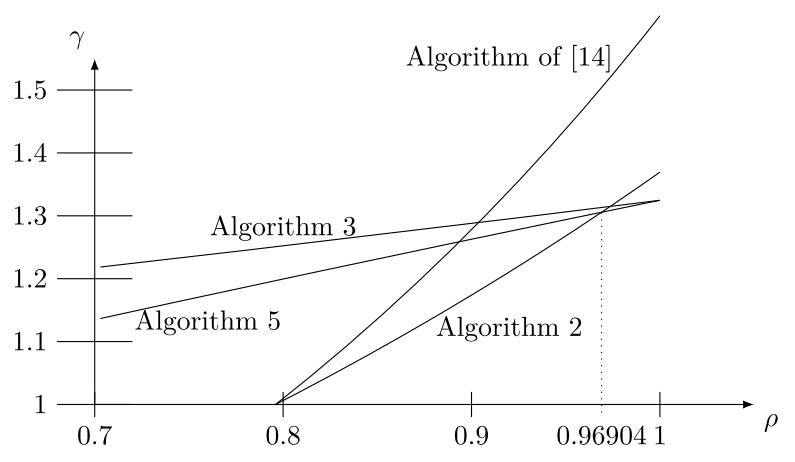

Fig. 1. Evaluation of complexities for different methods.

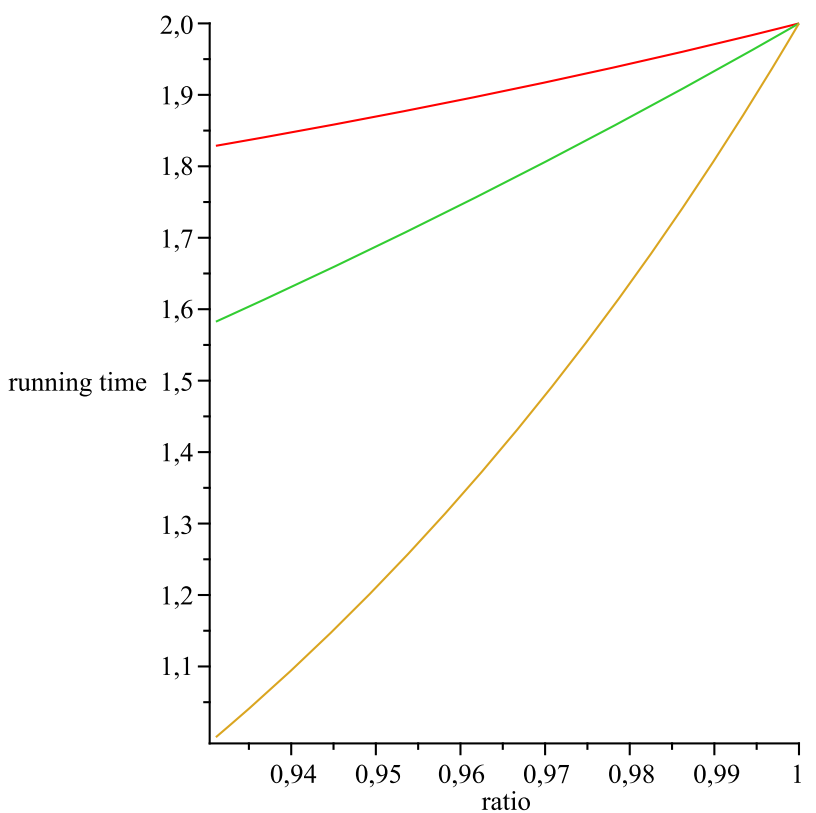

Fig. 2. Comparison between the algorithm of [20] for MAX-2-SAT (upper curve), the algorithms for MAX SAT (intermediate curve) and MAX-2-SAT (lower curve) that will be given in Section 4 .

uses roughly the same technique as in [14] (leading to Algorithm 2 in Fig. 1) while the second one uses a different approach consisting of splitting the instance in "small" sub-instances (Algorithm 3 in Fig. 1). In Section 3, we further improve these results for some ratios using another technique consisting of approximately pruning a search tree (Algorithm 5 in Fig. 1). Note that Fig. 1 is drawn using $\alpha=0.796$ as the best polynomial time approximation ratio, but a figure of similar shape would follow with other possible values of $\alpha$. In these sections, we also show that similar results can be derived for FPT approximation algorithms where, given a ratio $\rho$ and an integer $k$, one has either to output a solution that satisfies at least $\rho k$ clauses or to assert that no solution satisfies (at least) $k$ clauses.

All these results deal with complexity depending on the number of clauses. In Section 4, we consider complexity depending on the number of variables and improve the results of [20] (see Fig. 2) by giving for any ratio $\rho \leq 1$ a $\rho$-approximate algorithm that is (1) deterministic, (2) valid for MAX SAT (no restriction on the clauses length) and (3) with a much smaller running time (for any ratio $\rho$ ). We conclude the article in Section 5 where we also briefly discuss the MIN SAT problem.

\section{First results}

We provide in this section two first improvements of the result given in [14]. The first one, given in Section 2.1, uses the same idea as [14] while the second one uses a completely different technique and achieve improved running times (for some approximation ratios) by splitting the initial instance in sub-instances of smaller size. 


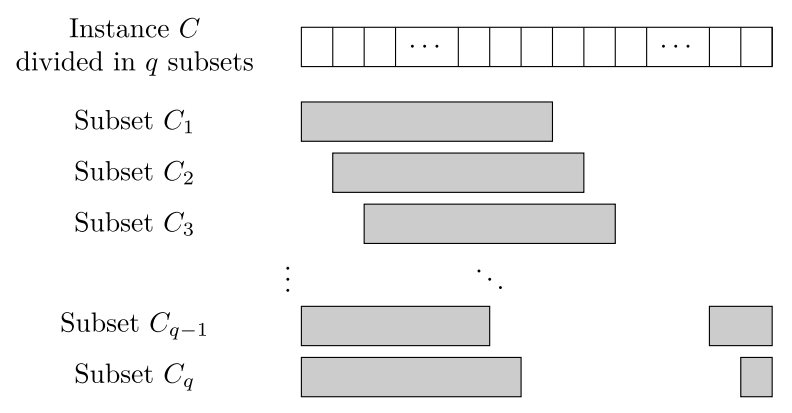

Fig. 3. Forming the $q$ subsets of clauses.

\subsection{Using a better parameterized algorithm}

In this section we briefly mention that the same technique as in [14] leads to an improved result when we build the search tree according to the algorithm from [10] instead of the search tree presented in Section 1. We so derive the following algorithm, that is strictly better than the one of [14] (see Fig. 1 in Section 1).

Algorithm 2. Build a search-tree as the parameterized algorithm of [10] does. ${ }^{3}$ Stop the development of this tree at each node where at least $(m(\rho-\alpha) /(1-\alpha))$ clauses are satisfied (recall that $\alpha$ is the best known polynomial approximation ratio for MAX SAT), or when the instance is empty. For each leaf of the so-pruned tree, apply a polynomial $\alpha$-approximation algorithm to complete the assignment of the remaining variables; thus, each leaf of the tree corresponds to a complete truth assignment. Return the assignment satisfying the largest number of clauses.

Proposition 1. For any $\rho$ such that $\alpha \leq \rho \leq 1$, Algorithm 2 achieves approximation ratio $\rho$ in time $0^{*}\left(1.3695^{m(\rho-\alpha) /(1-\alpha)}\right)$.

Proof. Consider first the running time. The parameterized algorithm of [10] builds a search tree where the worst case recurrence relation is $T(k) \leq 2 T(k-3)+2 T(k-7)$, where the parameter $k$ is the number of satisfied clauses, leading to a global complexity of $O^{*}\left(1.3695^{k}\right)$. Here, we build this tree and stop the construction in each leave where $m(\rho-\alpha) /(1-\alpha)$ clauses are satisfied. This leads to a running time of $O^{*}\left(1.3695^{m(\rho-\alpha) /(1-\alpha)}\right)$.

We now handle the approximation ratio. First, if the number $|\mathrm{OPT}|$ of clauses satisfied by an optimal solution OPT is less than $m(\rho-\alpha) /(1-\alpha)$, then Algorithm 2 obviously finds an optimum solution. Otherwise, let us consider the branch of the branching tree where the leaf corresponds to a partial optimal truth assignment satisfying clauses in OPT. Denote by $k_{0}$ the number of clauses satisfied in this leaf $\left(k_{0} \geqslant m(\rho-\alpha) /(1-\alpha)\right)$, i.e. by the partial assignment corresponding to this leaf. Using this assignment, we get a resulting instance in which it is possible to satisfy $|\mathrm{OPT}|-k_{0}$ clauses (because the optimal assignment satisfies $|\mathrm{OPT}|$ clauses). Consequently, the $\alpha$-approximation algorithm called by Algorithm 2 will satisfy at least $\alpha\left(|\mathrm{OPT}|-k_{0}\right)$ more clauses. So, finally, at least:

$$
\begin{aligned}
k_{0}+\alpha\left(|\mathrm{OPT}|-k_{0}\right) & =k_{0}(1-\alpha)+\alpha|\mathrm{OPT}| \geqslant m(\rho-\alpha)+\alpha|\mathrm{OPT}| \\
& \geqslant|\mathrm{OPT}|(\rho-\alpha)+\alpha|\mathrm{OPT}|=\rho|\mathrm{OPT}|
\end{aligned}
$$

clauses will be satisfied.

\subsection{Splitting the clauses}

In [8], it is shown that a generic method can give interesting moderately exponential approximation algorithms if applied in (maximization) problems satisfying some hereditary property (a property is said to be hereditary if for any set $A$ satisfying this property, and any $B \subset A, B$ satisfies this property too). MAX SAT can be seen as searching for a maximum subset of clauses satisfying the property "can be satisfied by a truth assignment", and this property is clearly hereditary. Therefore, we can adapt the splitting method introduced in [8] to transform any exact algorithm into a $\rho$-approximation algorithm, for any rational $\rho$, and with running time exponentially increasing with $\rho$.

Algorithm 3. Let $p, q$ be two integers such that $\rho=p / q$. Split the set of clauses into $q$ pairwise disjoint subsets $A_{1}, \cdots, A_{q}$ of size $m / q$ (at most $\lceil m / q\rceil$ if $m / q$ is not an integer). Then, consider the $q$ subsets $C_{i}=A_{i} \cup A_{i+1} \cup \ldots \cup A_{i+p-1}$ (if the index is larger than $q$, take it modulo $q$ ) for $i=1, \cdots, q$ (see Fig. 3). On each subset, apply some exact algorithm for MAX sat. Return the best truth assignment among them as solution for the whole instance.

\footnotetext{
3 Note that we use only the search-tree of the algorithm of [10] (in particular, not the initial kernelization in it), so that at least one branch of the tree corresponds to a partial optimal assignment.
} 
Proposition 2. Given an exact algorithm for MAX SAT running in time $O^{*}\left(\gamma^{m}\right)$, Algorithm 3 achieves approximation ratio $\rho$ in time $O^{*}\left(\gamma^{\rho m}\right)$.

Proof. Algorithm 3 calls $q$ times an exact algorithm (whose running time is $O^{*}\left(\gamma^{m}\right)$ ). Then the bound of the running time easily follows from the fact that each subset $C_{i}$ contains at most $p\lceil m / q\rceil \leq \rho m+p$ clauses.

For the approximation ratio, note first that if we restrict an instance with a set $C$ of clauses to a new instance with a new set $C^{\prime} \subset C$ of clauses, then an optimal solution for $C^{\prime}$ satisfies at least the same amount of clauses in $C^{\prime}$ than an optimal solution for $C$ (in other words, the restriction of any solution for $C$ to $C^{\prime}$ is feasible for $\left.C^{\prime}\right)$, i.e., $\left|\mathrm{OPT}(C) \cap C^{\prime}\right| \leqslant\left|\mathrm{OPT}\left(C^{\prime}\right)\right|$. In particular, for $i=1, \ldots, q,\left|\mathrm{OPT}\left(C_{i}\right)\right| \geqslant\left|\mathrm{OPT}(C) \cap C_{i}\right|$.

Now, note that by construction of the $C_{i}$ 's, we easily see that each clause appears in exactly $p$ among the $q$ subsets $C_{1}, C_{2}, \ldots, C_{q}$, and this holds in particular for any clause in OPT. Thus, $\sum_{i=1}^{q}\left|\mathrm{OPT}(C) \cap C_{i}\right|=p \times|\mathrm{OPT}(C)|$. By the discussion above, $\sum_{i=1}^{q}\left|\mathrm{OPT}\left(C_{i}\right)\right| \geqslant p \times|\mathrm{OPT}(C)|$. Since $\sum_{i=1}^{q}\left|\mathrm{OPT}\left(C_{i}\right)\right| \leqslant q \times \max _{i=1}^{q}\left|\mathrm{OPT}\left(C_{i}\right)\right|$, then $\max _{i=1}^{q}\left|\mathrm{OPT}\left(C_{i}\right)\right| \geqslant \frac{p}{q}|\mathrm{OPT}(C)|$.

Note that if we consider an FPT algorithm working in time $O^{*}\left(\gamma^{k}\right)$, using it in Algorithm 3 with parameter $\rho k$ (instead of an exact one) leads to a $\rho$ approximate FPT algorithm working in time $O^{*}\left(\gamma^{\rho k}\right)$. Also, it is worth noticing that Algorithm 3 is faster than Algorithm 2 for ratios close to 1 (see Fig. 1 in Section 1).

\section{Approximate pruning of the search tree}

Informally, the idea of an approximate pruning of the search tree is based upon the fact that, if we seek, say, a 1/2-approximation for a maximization problem, then when a search-tree based algorithm selects a particular datum $d$ for inclusion in the solution, one may remove one other datum $d^{\prime}$ from the instance (without, of course, including it in the solution). At worst, $d^{\prime}$ is part of an optimal solution and is lost by our solution. Thus, globally, the number of data in an optimum solution is at most two times the number of data in the built solution. On the other hand, with the removal of $d^{\prime}$, the size of the surviving instance is reduced not by 1 (due to the removal of $d$ ) but by 2 .

This method can be adapted to MAX SAT in the following way: revisit Algorithm 1 and recall that its worst case with respect to $m$ is to branch on a $(1,2)$-literal and to fix 1 (satisfied) clause on the one side and 2 (satisfied) clauses on the other side. If we decide to also remove 1 more clause (arbitrarily chosen) in the former case and 2 more clauses (arbitrarily chosen) in the latter one, this leads to a running time $T(m)$ satisfying $T(m) \leqslant T(m-2)+T(m-4)$, i.e., $T(m) \leqslant 0^{*}\left(1.27^{m}\right)$. Since in the branches we have satisfied at least $s \geq 1$ clause (resp., $s \geq 2$ clauses) while the optimum satisfies at most $s+1$ clauses (resp., $s+2$ clauses), we get an approximation ratio 0.5 .

This basic principle is not sufficient to get an interesting result for MAX SAT, but it can be improved as follows. Let us consider the left branch where the $(1,2)$-literal is set to true, satisfying a clause $C_{1}$. Instead of throwing away one other clause, we pick two clauses $C_{2}$ and $C_{3}$ such that $C_{2}$ contains a literal $\ell$ and $C_{3}$ contains the literal $\neg \ell$, and we remove these two clauses. Any truth assignment satisfies either $C_{2}$ or $C_{3}$, meaning that in this branch we will satisfy at least 2 clauses ( $C_{1}$ and one among $C_{2}$ and $C_{3}$ ), while at worst the optimum will satisfy these three clauses. In the other branch where 2 clauses are satisfied, we pick two pairs of clauses containing opposite literals and we remove them. This trick improves both the approximation ratio and the running time: now we have an approximation ratio $2 / 3$ (2 clauses satisfied among 3 clauses removed in one branch, 4 clauses satisfied among 6 clauses removed in the other branch), and the running time satisfies $T(m) \leqslant T(m-3)+T(m-6)$, i.e., $T(m)=O^{*}\left(1.17^{m}\right)$.

In what follows, we generalize the ideas sketched above in order to work for any ratio $\rho \in \mathbb{Q}$.

Algorithm 4. Let $p$ and $q$ be two integers such as $\frac{p}{q}=\frac{\rho-1}{1-2 \rho}$. We build the search tree and, on any of its nodes, we count the number of satisfied clauses from the root (we do not count here the clauses that have been arbitrarily removed). Each time we reach a multiple of $q$, we pick $p$ pairs of clauses with opposite literals and we remove them from the remaining sub-instance. When such a sub-instance on a node is empty, we arbitrarily assign a value on any still unassigned variable. Finally, we return the best truth assignment so constructed.

Note that it might be the case that at some point it is impossible to find $p$ pairs of clauses with opposite literals. But this means that (after removing $q<p$ pairs) each variable appears only positively or only negatively, and the remaining instance is easily solvable in linear time.

Theorem 1. Algorithm 4 runs in time $O^{*}\left(1.618^{m(2 \rho-1)}\right)$ and satisfies at least $\rho \cdot|O P T|$ clauses.

Proof. Consider the leaf where the variables are set like in an optimum solution. In this leaf, assume that the number of satisfied clauses is $s \times q+s^{\prime}$ (where $s^{\prime}<q$ ); again, we do not count the clauses that have been arbitrarily removed. Then, the algorithm has removed $s \times 2 p$ clauses arbitrarily, among which at least $s \times p$ are necessarily satisfied. In the worst case, the $s \times p$ other clauses are in OPT; hence, $|\mathrm{OPT}| \leqslant 2 s p+s q+s^{\prime}$. So, the approximation ratio of Algorithm 4 is at least: $\left(s q+s p+s^{\prime}\right) /\left(s q+2 s p+s^{\prime}\right) \geq \rho$. 
Table 1

Running times for the algorithm of [10] and Algorithm 5.

\begin{tabular}{|c|c|c|}
\hline Case & {$[10]$} & Algorithm 5 \\
\hline $4.0 \mathrm{a})$ & $T(m)=T(m-1)+T(m-5)$ & $T(m)=T(m-1-\chi)+T(m-5-4 \chi)$ \\
\hline $4.0 \mathrm{~b})$ & $T(m)=T(m-1)+T(m-7)+T(m-10)$ & $T(m)=T(m-1-\chi)+T(m-7-6 \chi)+T(m-10-9 \chi)$ \\
\hline 4.1 & $T(m)=2 T(m-3)$ & $T(m)=2 T(m-3-3 \chi)$ \\
\hline 4.2 & $T(m)=T(m-2)+T(m-3)$ & $T(m)=T(m-2-2 \chi)+T(m-3-2 \chi)$ \\
\hline 4.3 & $T(m)=2 T(m-6)+T(m-2)$ & $T(m)=2 T(m-6-6 \chi)+T(m-2-2 \chi)$ \\
\hline 4.4 & $T(m)=T(m-3)+T(m-2)$ & $T(m)=T(m-3-3 \chi)+T(m-2-2 \chi)$ \\
\hline 4.5 & Same as for 4.3 & \\
\hline 4.6 & Same as for 4.4 & \\
\hline 4.7 & $T(m)=2 T(m-5)$ & $T(m)=2 T(m-5-5 \chi)$ \\
\hline 4.8 & $T(m)=2 T(m-5)+2 T(m-7)$ & $T(m)=2 T(m-5-5 \chi)+2 T(m-7-6 \chi)$ \\
\hline 4.9 a) & Same as for 4.1 & \\
\hline $4.9 \mathrm{~b})$ & Same as for $4.0 \mathrm{a}$ ) & \\
\hline 4.10 & $T(m)=T(m-1)+1$ & $T(m)=T(m-1)+1$ \\
\hline 4.11 a) & $T(m)=2 T(m-4)$ & $T(m)=2 T(m-4-4 \chi)$ \\
\hline $4.11 \mathrm{~b})$ & Same as for $4.0 \mathrm{a}$ ) & \\
\hline 4.12 a) & Same as for $4.0 \mathrm{a}$ ) & \\
\hline $4.12 \mathrm{~b})$ & $T(m)=2 T(m-8)+T(m-1)$ & $T(m)=2 T(m-8-7 \chi)+T(m-1-\chi)$ \\
\hline
\end{tabular}

We now estimate the running time of Algorithm 4. For each node $i$ of the tree, denote by $m_{i}$ the number of clauses left in the surviving sub-instance of this node, by $z_{i}$ the number of satisfied clauses from the root of the tree (we do not count the clauses that have been arbitrarily removed) and set $t_{i}=m_{i}-(2 p / q)\left(z_{i} \bmod q\right)$.

For the root of the tree, $z_{i}=0$ and therefore $t_{i}=m$. Let $i$ be a node with two children $j$ (at least one clause satisfied) and $g$ (at least two clauses satisfied). Let us examine quantity $t_{j}$ when exactly one clause is satisfied. In this case, $z_{j}=z_{i}+1$. On the other hand: i) If $z_{j} \bmod q \neq 0$, then we have not reached the threshold necessary to remove the $2 p$ clauses. Then, $m_{j}=m_{i}-1$ and $t_{j}=m_{j}-2 p / q\left(z_{j} \bmod q\right)=m_{i}-1-2 p / q\left(\left(z_{i} \bmod q\right)+1\right)=t_{i}-1-2 p / q$. If $z_{j} \bmod q=0$, then $z_{i} \bmod q=$ $q-1$ and the threshold has been reached; so $2 p$ clauses have been removed. Then, $m_{j}=m_{i}-1-2 p, t_{j}=m_{j}=m_{i}-1-2 p$ and $t_{i}=m_{i}-2 p / q(q-1)=m_{i}-2 p+2 p / q$. Finally, $t_{j}=t_{i}-1-2 p / q$. Therefore, in both cases i) and ii), $t_{j} \leqslant t_{i}-1-2 p / q$. Of course, by a similar argument, if we satisfy $g$ clauses, then the quantity $t_{i}$ is reduced by $g(1+2 p / q)$. This leads to a running time $T(t) \leqslant T(t-1-2 p / q)+T(t-2-4 p / q)$ and hence $T(t)=1.618^{t /(1+2 p / q)}$. Since initially $t=m$, we get $T(m)=1.618^{m /(1+2 p / q)}$. Taking into account that $p / q=(\rho-1) /(1-2 \rho)$, we get immediately $1 /(1+2 p / q)=2 \rho-1$.

Algorithm 4 can be improved if instead of using the simple branching rule in the tree, the more involved case analysis of [10] is used. As already noted in Section 2.1, we use only the search-tree of the algorithm of [10], that ensures that at least one branch of the tree corresonds to a partial optimal assignment. This derives the following algorithm.

Algorithm 5. Let $p$ and $q$ be two integers such as $\frac{p}{q}=\frac{\rho-1}{1-2 \rho}$. Build the search-tree of [10] and, on each node of it, count the number of satisfied clauses from the root. Each time a multiple of $q$ is reached, pick $p$ pairs of clauses with opposite literals and remove them from the resulting sub-instance. Return the best truth assignment so constructed.

To estimate the running time of Algorithm 5, we use nearly the same analysis as in [10]. The only difference is that, at each step of the search tree, [10] counts without distinction the satisfied and the unsatisfied clauses (clauses that became empty), whereas we have to make a difference in the complexity analysis: a satisfied clause reduces the quantity $t$ by $1+2 p / q$ in Algorithm 5, while an unsatisfied clause reduces it by only 1 .

By an exhaustive comparative study between the cases analyzed in [10] and Algorithm 5, it can be shown that for any $\rho$ the worst case is always reached by the case (noted by 4.2 in [10]) $T(m)=T(m-2)+T(m-3)$, that becomes $T(m)=T(m-2-2 \chi)+T(m-3-2 \chi)$ with $\chi=2 p / q$ in the analysis of Algorithm 5 .

Indeed, in case 4.2 of [10] ("there is (2,2)-literal $x$ that occurs at least once as a unit clause"), a branching is done on the variable $x$. On the one side, 2 clauses are satisfied while, on the other side, 2 are satisfied and 1 becomes empty and thus it is removed from the instance. For [10], this leads to a complexity $T(m) \leqslant T(m-2)+T(m-3)$. For Algorithm 5, this gives $T(m) \leqslant T(m-2-4 p / q)+T(m-3-4 p / q)$. To simplify these results, set $\chi=2 p / q$. Then $T(m) \leqslant T(m-2-2 \chi)+$ $T(m-3-2 \chi)$, which leads to $T(m)=O^{*}\left(\gamma^{m}\right)$ with $\gamma$ the largest real solution of the equation $\gamma^{2 \chi+3}-\gamma-1=0$.

For the other cases, a comparative study between the algorithm of [10] and Algorithm 5 is summarized in Table 1. Its third column gives equations whose largest real solutions are the worst case running times for Algorithm 5 .

Depending on the ratio $\rho$ we seek, the running time of the algorithm is given by the worst case of all the cases given in Table 1 . However, one can show that for any $\rho$ the worst case is always reached by the case 4.2. Let us show an example (the other cases are similar). Consider the equations $f_{4.2}(X)=X^{2 \chi+3}-X-1=0$ and $f_{4.0}(X)=X^{4 \chi+5}-X^{3} \chi+4-1=0$. The largest real solution of the former is always larger than the largest real solution of the latter one. Indeed, let $\chi$ be any positive value. Remark first that $f_{4.0}^{\prime}(X)=(4 \chi+5) X^{4} \chi+4-(3 \chi+4) X^{3} \chi+3>0$; hence, function $f_{4.0}$ is increasing with 


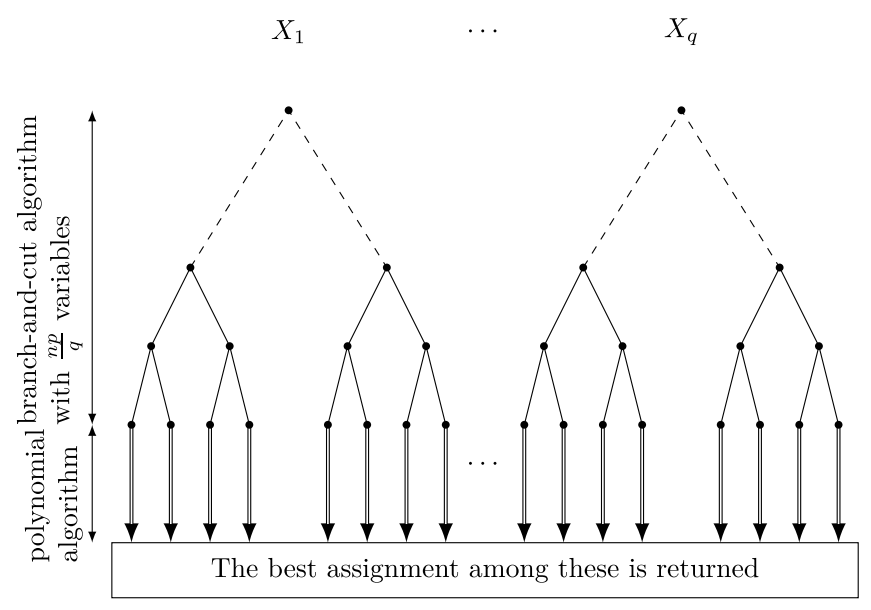

Fig. 4. Illustration of Algorithm 6 .

$X>1$. What we now need to show is that if $f_{4.2}(X)=0$, then $f_{4.0}(X) \geqslant 0$ (this means that the zero of $f_{4.0}$ is before that of $\left.f_{4.2}\right)$ :

$$
\begin{aligned}
f_{4.2}(X)=0 & \Leftrightarrow X^{2 \chi+3}=X+1 \quad \Leftrightarrow \quad X^{3 \chi+4}=X^{\chi+2}+X^{\chi+1} \\
& \Leftrightarrow X^{4 \chi+5}=X^{2 \chi+3}+X^{2 \chi+2} \Leftrightarrow X^{4 \chi+5}=X^{2 \chi+2}+X+1 \\
& \Rightarrow X^{4 \chi+5}-X^{3 \chi+4}-1=X^{2 \chi+2}+X-X^{\chi+2}-X^{\chi+1} \\
& \Rightarrow f_{4.0}(X)=X^{2 \chi+2}+X-X^{\chi+2}-X^{\chi+1} \\
& \Rightarrow f_{4.0}(X)=\left(X^{\chi+1}-1\right)\left(X^{\chi+1}-X\right) \geqslant 0
\end{aligned}
$$

and the result follows.

Now, the claim of Theorem 1 dealing with the approximation ratio of Algorithm 4 identically applies also for Algorithm 5 . Putting all the above together, the following theorem holds.

Theorem 2. For any $\rho<1$, Algorithm 5 achieves approximation ratio $\rho$ on MAX SAT with running time $T(m)=O^{*}\left(\gamma^{m}\right)$, where $\gamma$ is the largest real solution of the equation $X^{2 \alpha+3}-X-1=0$ and $\alpha=\frac{2 \rho-2}{1-2 \rho}$.

It is very well-known that, in every MAX SAT-instance, at least $m / 2$ clauses can be always greedily satisfied. So for any such formula, $|\mathrm{OPT}| \geqslant m / 2$. Hence, any algorithm with running time function of $m$ is a parameterized algorithm for MAX SAT. This however may lead to uninteresting results (its running time may be worse than that of the parameterized algorithm of [10]), but we can improve this. Indeed, we can show that the pruning method just described can be directly applied to the parameterized algorithm of [10] for the achievement of the following parameterized approximation result.

Proposition 3. For any $\rho<1$, MAX SAT is approximable within ratio $\rho$ in time $O^{*}\left(1.3695^{(2 \rho-1) k}\right)$, where $k$ is the maximum number of satisfied clauses in the instance.

\section{Splitting the variables}

In this section, we present two algorithms that approximate MAX SAT within any approximation ratio smaller than 1, and with a computation time depending on $n$ (the number of variables). As mentioned in the introduction, Hirsch [20] devises for any $\epsilon>0$ and any $k \geq 2$ a randomized algorithm that find with high probability a $(1-\epsilon)$ approximation for MAX-k-SAT in time $O^{*}\left(\left(2-c_{\epsilon, k}\right)^{n}\right)$ where $c_{\epsilon, k}=2 \epsilon /\left(k(1+\epsilon)\right.$ ) (note that $c_{\epsilon, k} \rightarrow 0$ when $k \rightarrow \infty$, so this does not give a complexity $c^{n}$ with $c<2$ for MAX SAT). For MAX-2-SAT, the complexity is improved down to $0^{*}\left((2-3 \epsilon /(1+3 \epsilon))^{n}\right)$.

As we will see, the first algorithm of this section (Algorithm 6 - see Fig. 4 for an illustration) improves these results. It builds several trees. Then, in each of them, as for Algorithm 2 in Section 2.1, it cuts the tree at some point and completes variables' assignment using a polynomial approximation algorithm.

Algorithm 6. Let $p$ and $q$ be two integers such that $p / q=(\rho-\alpha) /(1-\alpha)$. Build $q$ subsets $X_{1}, \cdots, X_{q}$ of variables, each one containing roughly $p / q \times n$ variables, where each variable appears in exactly $p$ subsets (as in Algorithm 3 in Section 2.2 ). For each subset $X_{i}$, construct a complete search tree, considering only the variables in the subset (i.e., the depth of each of 
these trees is exactly $\left.\left|X_{i}\right| \simeq p / q \times n\right)$. For each of the leaves of these trees, run a polynomial time algorithm guaranteeing a ratio $\alpha$ on the surviving sub-instance. Return the best truth assignment among those built.

Each of the trees built by Algorithm 6 is a binary tree and has depth roughly $p n / q$ (more precisely, at most $p n / q+p$ ). So its running time is $O^{*}\left(2^{n p / q}\right)$. Note also that, on each of these trees, at least one leaf is a partial assignment of an optimal (global) truth assignment. We will call such a leaf an optimal leaf.

Lemma 1. At least one of optimal leaf has at least $\frac{p}{q} \times|O P T|$ satisfied clauses (before applying the polytime approximation algorithm).

Proof. Remark that every clause $C_{i}$ in OPT contains at least one true literal; pick one of them from each clause $C_{i}$ and denote the variable corresponding to this literal by $\operatorname{Var}\left(C_{i}\right)$. Let, for each variable $x, C(x)$ be the set of clauses from OPT for which $x$ or $\neg x$ is the picked literal, i.e., $\forall x \in X, C(x)=\left\{C_{i} \in\right.$ OPT $\left./ \operatorname{Var}\left(C_{i}\right)=x\right\}$. Based upon this, OPT $=\bigcup_{x \in X} C(x)$.

In the tree obtained on the set $X_{i}$, denote by $\Lambda_{i}$ the set of satisfied clauses on some optimal leaf and set $\lambda_{i}=\left|\Lambda_{i}\right|$. Then, $\bigcup_{x \in X_{i}} C(x) \subseteq \Lambda_{i}$ and, by construction, $\forall i, j, C\left(x_{i}\right) \cap C\left(x_{j}\right)=\emptyset$. We so have:

$$
\begin{aligned}
& \lambda_{i} \geqslant \sum_{x \in X_{i}}|C(x)| \\
& \sum_{i=1}^{q} \lambda_{i} \geqslant \sum_{i=1}^{q} \sum_{x \in X_{i}}|C(x)|
\end{aligned}
$$

As every $x$ belongs to exactly $p$ subsets among the $q$ sets $X_{i}$, it holds that:

$$
\sum_{i=1}^{q}\left|\lambda_{i}\right| \geqslant p \times \sum_{x \in X}|C(x)|=p \times|\mathrm{OPT}|
$$

From (1), it is immediately derived that:

$$
\max _{i=1}^{q}\left|\lambda_{i}\right| \geqslant \frac{1}{q} \sum_{i=1}^{q}\left|\lambda_{i}\right| \geqslant \frac{p}{q} \times \sum_{x \in X}|C(x)|=\frac{p}{q} \times|\mathrm{OPT}|
$$

that concludes the proof.

Proposition 4. Algorithm 6 achieves approximation ratio $\rho$.

Proof. By Lemma 1, among all the optimal leaves, at least one satisfies $\lambda \geqslant \frac{p}{q} \times|\mathrm{OPT}|$ clauses. As an optimal leaf corresponds to an optimal truth assignment, it is possible to complete this assignment into an optimal (global) solution. In other words, there exist $|\mathrm{OPT}|-\lambda$ remaining clauses that become true on the surviving sub-instance. If the polynomial algorithm called by Algorithm 6 achieves approximation ratio $\alpha$, it will compute a solution that satisfies at least $\alpha \times(|\mathrm{OPT}-| \lambda \mid)$ clauses Hence, the number of satisfied clauses will be at least:

$$
|\lambda|+\alpha \times(|\mathrm{OPT}-| \lambda \mid)=\alpha|\mathrm{OPT}|+(1-\alpha) \lambda \geqslant \alpha|\mathrm{OPT}|+(1-\alpha) \frac{p}{q}|\mathrm{OPT}|
$$

that leads to an approximation ratio of $\alpha+(1-\alpha) \frac{p}{q}=\rho$.

Putting all the above together, the following theorem holds.

Theorem 3. Algorithm 6 achieves ratio $\rho$ in time $O^{*}\left(2^{n(\rho-\alpha) /(1-\alpha)}\right)$, for any $\rho \leq 1$.

Algorithm 6 is both deterministic and valid for MAX SAT. Moreover in [20] the best running time is obtained for the restricted problem MAX-2-SAT for which a $\rho=(1-\epsilon)$-approximate solution is found in time $0^{*}\left((2-3(1-\rho) /(4-3 \rho))^{n}\right)$. Interestingly enough, $(2-3(1-\rho) /(4-3 \rho))$ is greater than $2^{(\rho-\alpha) /(1-\alpha)}$ (with $\left.\alpha=0.796\right)$ for any $\rho<1$. Finally, note that for MAX-2-SAT one can use Theorem 3 with the best polynomial time approximation ratio known for this problem, i.e., $\alpha=0.931$ [17] (note that MAX-2-SAT is not approximable in polynomial time within ratio 0.955 unless $\mathbf{P}=\mathbf{N P}$ [19]). See Fig. 2 in Section 1 for a comparison of running times.

Algorithm 6 builds a full search tree on each subset of variables. In particular, when the ratio sought $\rho$ tends to 1, the basis of the exponent in the complexity tends to 2. Then, one might ask the following question: suppose that there is an exact algorithm solving MAX SAT in $O^{*}\left(\gamma^{n}\right)$ (for some $\gamma<2$ ), is it possible to find a $\rho$ approximation algorithm in time $0^{*}\left(\gamma_{\rho}^{n}\right)$ where $\gamma_{\rho}<\gamma$ for some $\left.\left.\rho \in\right] \alpha, 1\right]$ ? For any $\left.\left.\rho \in\right] \alpha, 1\right]$ ? This kind of reduction from an approximate solution to an 
exact one would allow to take advantage of any possible improvement of the exact solution of MAX SAT, which is not the case in Algorithm 6. Note that finding an exact algorithm in time $0^{*}\left(\gamma^{n}\right)$ for some $\gamma<2$ is a famous open question for MAX SAT (cf. the strong exponential time hypothesis [21]) as well as for some other combinatorial problems. It has very recently received a positive answer for the Hamiltonian cycle problem in [4].

Indeed, we propose in what follows a $\rho$-approximation algorithms working in time $O^{*}\left(\gamma_{\rho}^{n}\right)$ with $\gamma_{\rho}<\gamma$ for any $\rho \in] \alpha, 1$. We first give a simple solution (Algorithm 7) that we improve later (Algorithm 8). Algorithm 7 moves in the same spirit as Algorithm 6 but, instead of building a full branching tree on $X_{i}$, calls an exact algorithm on the sub-instance induced by the set $X_{i}$.

Algorithm 7. Let $\rho \in \mathbb{Q}$ and $p$ and $q$ two integers such that $p / q=\rho$. Build $q$ subsets of variables, each one containing $p / q \times n$ variables (as in Algorithm 6). For each subset of variables $X_{i}$ :

a) Remove from the instance the variables not in $X_{i}$ and any empty clause.

b) Run the exact algorithm on the resulting sub-instance, thus obtaining a truth assignment for the variables in $X_{i}$.

c) Complete this assignment with arbitrary truth-values for the variables not in $X_{i}$.

Among all the truth assignments produced, return the one that satisfies the largest number of clauses in the whole instance.

In Algorithm 7, the exact algorithm called in step b) runs in time $0^{*}\left(\gamma^{\rho n}\right)$. Its approximation ratio is the one claimed in Lemma 1. Indeed, the exact algorithm satisfies at least the same amount of clauses as the optimal branching (for the global instance) would do. More precisely, for each $X_{i}$, and for any $x \in X_{i}$, the clauses containing $x$ (and in particular the clauses in $C(x)$ ) are not removed from the instance. The optimal branching would then satisfy at least $\sum_{x \in X_{i}}|C(x)|$ clauses and, obviously, the exact algorithm would satisfy even more. Hence, the following result holds.

Proposition 5. Algorithm 7 achieves approximation ratio $\rho$ in time $O^{*}\left(\gamma^{\rho n}\right)$, where $O^{*}\left(\gamma^{n}\right)$ is the running time of an exact algorithm for MAX SAT.

As one can see, in step c) of Algorithm 7, variables outside $X_{i}, i=1, \ldots, q$, are assigned arbitrarily, so, at worst their truth value may satisfy no additional clause. Note that one might want to use an approximation algorithm in the remaining instance as in Algorithm 6; however, the same analysis would not work since the exact solution obtained by the exact algorithm on the sub-instance might be completely different from the partial assignment of a global optimal solution. Nevertheless, we are able to propose an improvement by completing partial solutions in such a way that, roughly speaking, at least half of the remaining clauses are satisfied.

We now propose Algorithm 8, that is an improvement of Algorithm 7. For any $\rho \in] \alpha, 1]$, it achieves approximation ratio $\rho$ and runs in time $O^{*}\left(\gamma_{\rho}^{n}\right)$.

Algorithm 8. Let $p, q \in \mathbb{Q}$ be such that $p / q=2 \rho-1$. Build $q$ subsets of variables $X_{1}, \ldots, X_{q}$, each one containing $p / q \times n$ variables (as in Algorithm 6). For each $X_{i}$ run the following steps:

i) assign weight 2 to every clause containing only variables in $X_{i}$, and weight 1 to every clause containing at least one variable not in $X_{i}$;

ii) remove from the instance the variables not in $X_{i}$; remove empty clauses;

iii) solve exactly this MAX WEIGHTED SAT resulting instance, thus obtaining a truth assignment for the variables in $X_{i}$;

iv) complete the assignment with a greedy algorithm: for each $(i, j)$-literal, if $i>j$, then the literal is set to TRUE, else it is set to FALSE (and the instance is modified accordingly) and return the best among the truth-assignments soproduced.

Lemma 2. If there is a MAX SAT-algorithm working in time $0^{*}\left(\gamma^{n}\right)$, then the instances of MAX WEIGHTED SAT in Algorithm 8 can be solved with the same bound on the running time.

Proof. Note that the only weights assigned by Algorithm 8 are 1 and 2 . In such a weighted instance, we can add a new variable $x_{0}$ and replace each clause $c$ of weight 2 by three new clauses: $c, c \vee x_{0}$ and $c \vee \neg x_{0}$. Thus, if $c$ is satisfied, then it will count in the new instance as three satisfied clauses. Otherwise, exactly one of the three new clauses will be satisfied. Thus, the so-built instance of MAX SAT is equivalent to the initial MAX WEIGHTED SAT-instance built by Algorithm 8 .

Theorem 4. Algorithm 8 achieves approximation ratio $\rho$ in time $O^{*}\left(\gamma^{(2 \rho-1) n}\right)$, where $O^{*}\left(\gamma^{n}\right)$ is the running time of an exact algorithm for MAX SAT.

Proof. For the running time: we apply $q$ times an exact algorithm $O^{*}\left(\gamma^{n}\right)$ on instances of size $(2 \rho-1) n$. 


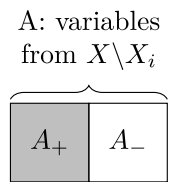

Clauses in OPT

Clauses not in OPT

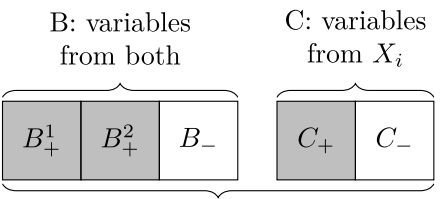

Part remaining of the instance after step 2, on which we apply the MAX WEIGHTED SAT algorithm

Fig. 5. Division of clauses according to a subset $X_{i}$ of variables.

For the approximation ratio, using the same notation as before, consider one particular literal in each clause satisfied by some optimum solution OPT, and let $C(x)$ be the subset of these clauses such that the picked literal is $x$ or $\neg x$. Then, as shown before, there exists a subset $X_{i}$ such that $\sum_{x \in X_{i}}|C(x)| \geqslant \frac{p}{q}|\mathrm{OPT}|$. Consider now such a $X_{i}$, and denote by (see Fig. 5): $A$ the subset of clauses containing only variables in $X \backslash X_{i}, A_{+}$(resp., $A_{-}$) the subset of clauses from $A$ that are in the optimum (resp., are not in the optimum); $B$ the subset of clauses containing at least one variable in $X_{i}$ and one variable in $X \backslash X_{i} ; B_{+}^{1}$ (resp., $B_{+}^{2}$ ) the subset of clauses from $B$ that are in the optimum and whose chosen variable $\operatorname{Var}(c)$ is in $X_{i}$ (resp., not in $X_{i}$ ); $B_{-}$the subset of clauses from $B$ that are not in the optimum; $C$ the remaining clauses, i.e., the clauses that contain only variables in $X_{i}, C_{+}$(resp., $C_{-}$) the subset of clauses from $C$ that are in the optimum (resp., are not in the optimum). Note that when removing variables in $X \backslash X_{i}$, clauses in $A$ become empty, so the remaining clauses are exactly those in $B \cup C$. With these notations, OPT $=A_{+} \cup B_{+}^{1} \cup B_{+}^{2} \cup C_{+}$and $\sum_{x \in X_{i}}|C(x)|=\left|B_{+}^{1}\right|+\left|C_{+}\right|$. Then, for the chosen $X_{i}$ :

$$
\left|B_{+}^{1}\right|+\left|C_{+}\right| \geqslant \frac{p}{q}|\mathrm{OPT}|=\frac{p}{q}\left(\left|A_{+}\right|+\left|B_{+}^{1}\right|+\left|B_{+}^{2}\right|+\left|C_{+}\right|\right)
$$

With respect to step iii) of Algorithm 8, denote by $B_{1}$ the subset of satisfied clauses from $B$ and by $C_{1}$ the subset of satisfied clauses from $C$. As OPT is a particular solution of weight $\left|B_{+}^{1}\right|+2\left|C_{+}\right|$for this weighted sat problem, we have:

$$
\left|B_{1}\right|+2\left|C_{1}\right| \geqslant\left|B_{+}^{1}\right|+2\left|C_{+}\right|
$$

The greedy algorithm in step iv) will satisfy at least half of the remaining clauses containing at least one literal from $X \backslash X_{i}$, i.e., the set $\left(B \backslash B_{1}\right) \cup A$. Finally, the number of satisfied clauses is at least:

$$
\begin{aligned}
\left|B_{1}\right|+\left|C_{1}\right|+\frac{|B|-\left|B_{1}\right|+|A|}{2} & =\left|C_{1}\right|+\frac{\left|B_{1}\right|}{2}+\frac{|B|}{2}+\frac{|A|}{2} \geqslant \frac{(3)}{2}+\left|B_{+}^{1}\right| \\
& \geqslant\left|B_{+}^{1}\right|+\left|C_{+}\right|+\frac{|B|}{2}+\frac{|A|}{2}+\frac{\left|A^{+}\right|}{2}
\end{aligned}
$$

So, the approximation ratio achieved is at least:

$$
\frac{\left|B_{+}^{1}\right|+\left|C_{+}\right|+\frac{\left|B_{+}^{2}\right|+\left|A_{+}\right|}{2}}{\left|B_{+}^{1}\right|+\left|C_{+}\right|+\left|B_{+}^{2}\right|+\left|A_{+}\right|}=\frac{1}{2}\left(1+\frac{\left|B_{+}^{1}\right|+\left|C_{+}\right|}{\left|B_{+}^{1}\right|+\left|C_{+}\right|+\left|B_{+}^{2}\right|+\left|A_{+}\right|}\right) \geqslant \frac{1}{2}\left(1+\frac{p}{q}\right)=\frac{q+p}{2 q}=\rho
$$

that completes the proof.

For instance, suppose that MAX SAT is solvable in $O^{*}\left(1.657^{n}\right)$, which is the running time to solve Hamiltonian cycle in [4]. Then Algorithm 8 achieves a 0.9 -approximation in time $O^{*}\left(1.576^{n}\right)$ while Algorithm 6 achieves the same ratio in time $O^{*}\left(1.703^{n}\right)$.

\section{Discussion}

We have proposed in this paper several algorithms that constitute a kind of "moderately exponential approximation schemata" for MAX SAT. They guarantee approximation ratios that are unachievable in polynomial time unless $\mathbf{P}=\mathbf{N P}$, or even in time $2^{m^{1-\epsilon}}$ under the exponential time hypothesis. To obtain these schemata, several techniques have been used coming either from the polynomial approximation or from the exact computation. Furthermore, Algorithm 8 in Section 4 is a kind of polynomial reduction between exact computation and moderately exponential approximation transforming exact algorithms running on "small" sub-instances into approximation algorithms guaranteeing good ratios for the whole instance. We think that research in moderately exponential approximation is an interesting research issue for overcoming limits posed to the polynomial approximation due to the strong inapproximability results proved in the latter paradigm.

We conclude this paper with a word about another very well known optimum satisfiability problem, the MIN SAT problem that, given a set of variables and a set of disjunctive clauses, consists of finding a truth assignment that minimizes the 
number of satisfied clauses. A $\rho$-approximation algorithm for MIN SAT (with $\rho>1$ ) is an algorithm that finds an assignment satisfying at most $\rho$ times the minimal number of simultaneously satisfied clauses.

In [11] an approximability-preserving reduction between MIN VERTEX COVER and MIN SAT is presented transforming any $\rho$-approximation for the former problem into a $\rho$-approximation for the latter problem. This reduction can be used to translate any result on the MIN VERTEX COVER problem into a result on the MIN SAT, the number of vertices in the MIN VERTEX COVER instance being the number of clauses in the MIN SAT instance. For instance, the results from [8] for MIN VERTEX COVER lead to the following parameterized approximation result for MIN SAT: for every instance of MIN SAT and for any $r \in \mathbb{Q}$, if there exists a solution for MIN SAT satisfying at most $k$ clauses, it is possible to determine with complexity $0^{*}\left(1.28^{\text {rk }}\right)$ a $2-r$-approximation of it.

We also note that the method used in Algorithm 6 can be applied as well to MIN SAT with the following modification of the algorithm. Let $p, q \in \mathbb{Q}$ be such that $p / q=2 \rho-1$. Build $q$ subsets of variables, each one containing $p / q \times n$ variables. For each subset, construct a search tree, considering only the variables in the subset (the depth of the trees is $p / q \times n$ ). For each leaf of any of the so-built trees, use some polynomial algorithm with ratio $\alpha$ on the surviving sub-instance. Return the best of the truth assignments computed.

The complexity of the modification just described is the same as that of Algorithm 6, i.e., $O^{*}\left(2^{n(\alpha-\rho) /(\alpha-1)}\right)($ the best known ratio is $\alpha=2$ ), and a similar analysis derives an approximation ratio $\alpha-(\alpha-1) \frac{p}{q}=\rho$.

\section{References}

[1] G. Ausiello, P. Crescenzi, G. Gambosi, V. Kann, A. Marchetti-Spaccamela, M. Protasi, Complexity and Approximation. Combinatorial Optimization Problems and Their Approximability Properties, Springer-Verlag, Berlin, 1999.

[2] A. Avidor, I. Berkovitch, U. Zwick, Improved approximation algorithms for MAX NAE-SAT and MAX SAT, in: T. Erlebach, G. Persiano (Eds.), Proc. Workshop on Approximation and Online Algorithms, WAOA'05, in: Lecture Notes in Computer Science, vol. 3879, Springer-Verlag, 2006, pp. 27-40.

[3] R. Battiti, M. Protasi, Algorithms and heuristics for max-sat, in: D.Z. Du, P.M. Pardalos (Eds.), Handbook of Combinatorial Optimization, vol. 1, Kluwer Academic Publishers, 1998, pp. 77-148.

[4] A. Björklund, Determinant sums for undirected Hamiltonicity, in: Proc. FOCS'10, IEEE Computer Society, 2010, pp. $173-182$.

[5] A. Björklund, T. Husfeldt, M. Koivisto, Set partitioning via inclusion-exclusion, SIAM J. Comput. 39 (2) (2009) 546-563.

[6] N. Bourgeois, B. Escoffier, V.Th. Paschos, Efficient approximation of MIN coloring by moderately exponential algorithms, Inform. Process. Lett. 109 (16) (2009) 950-954.

[7] N. Bourgeois, B. Escoffier, V.Th. Paschos, Efficient approximation of MIN SET cover by moderately exponential algorithms, Theoret. Comput. Sci. 410 (21-23) (2009) 2184-2195.

[8] N. Bourgeois, B. Escoffier, V.Th. Paschos, Approximation of MAX INDEPENDENT SET, MIN VERTEX cover and related problems by moderately exponential algorithms, Discrete Appl. Math. 159 (17) (2011) 1954-1970.

[9] L. Cai, X. Huang, Fixed-parameter approximation: conceptual framework and approximability results, in: H.L. Bodlaender, M.A. Langston (Eds.), Proc. International Workshop on Parameterized and Exact Computation, IWPEC'06, in: Lecture Notes in Computer Science, vol. 4169, Springer-Verlag, 2006, pp. 96-108.

[10] J. Chen, I.A. Kanj, Improved exact algorithms for MAX SAT, Discrete Appl. Math. 142 (2004) 17-27.

[11] P. Crescenzi, R. Silvestri, L. Trevisan, To weight or not to weight: where is the question?, in: Proc. Israeli Symposium on Theory of Computing and Systems, ISTCS'96, IEEE, 1996, pp. 68-77.

[12] M. Cygan, L. Kowalik, M. Wykurz, Exponential-time approximation of weighted set cover, Inform. Process. Lett. 109 (16) (2009) 957-961.

[13] M. Cygan, M. Pilipczuk, Exact and approximate bandwidth, Theoret. Comput. Sci. 411 (40-42) (2010) 3701-3713.

[14] E. Dantsin, M. Gavrilovich, E.A. Hirsch, B. Konev, MAX SAT approximation beyond the limits of polynomial-time approximation, Ann. Pure Appl. Logic $113(2002) 81-94$.

[15] R.G. Downey, M.R. Fellows, C. McCartin, Parameterized approximation problems, in: H.L. Bodlaender, M.A. Langston (Eds.), Proc. International Workshop on Parameterized and Exact Computation, IWPEC'06, in: Lecture Notes in Computer Science, vol. 4169, Springer-Verlag, 2006 , pp. 121-129.

[16] B. Escoffier, V.Th. Paschos, A survey on the structure of approximation classes, Comput. Sci. Rev. 4 (1) (2010) 19-40.

[17] U. Feige, M.X. Goemans, Approximating the value of two prover proof systems, with applications to max 2sat and max dicut, in: Proc. Israeli Symposium on Theory of Computing and Systems, ISTCS'95, 1995, pp. 182-189.

[18] M. Fürer, S. Gaspers, S.P. Kasiviswanathan, An exponential time 2-approximation algorithm for bandwidth, in: Proc. International Workshop on Parameterized and Exact Computation, IWPEC'09, in: Lecture Notes in Computer Science, vol. 5917, Springer, 2009, pp. 173-184.

[19] J. Håstad, Some optimal inapproximability results, in: Proc. STOC'97, 1997, pp. 1-10.

[20] E.A. Hirsch, Worst-case study of local search for MAX-k-SAT, Discrete Appl. Math. 130 (2) (2003) $173-184$.

[21] R. Impagliazzo, R. Paturi, F. Zane, Which problems have strongly exponential complexity?, J. Comput. System Sci. 63 (4) (2001) $512-530$.

[22] D. Moshkovitz, R. Raz, Two query pcp with sub-constant error, in: Proc. FOCS'08, 2008, pp. 314-323. 